\title{
Abiotic stress and crop improvement: current scenario
}

\section{Opinion}

Abiotic stresses such as drought, salinity, heat and cold, are greatly affecting the plant growth and agricultural productivity and causes more than $50 \%$ of worldwide yield loss of major crops every year. Drought and low soil fertility are the major significant problem causes, among the abiotic stresses affecting crop production in developing countries. Crop loss to drought in the tropics has virtually exceeded 20 million tons of grain equivalent per year, or around 17 percent of wellwatered production, reaching up to 60 percent in severely affected regions such as southern Africa in 1991-92. ${ }^{1}$ Due to the deterioration of global climate, this becomes a worldwide exacerbated problem. Hence, it is very urgent to improve the qualities of the crop against multiple environmental stresses. ${ }^{2}$

Substantial progress has been made to unravel the molecular mechanisms of abiotic stress responses in plants by means of high throughput sequencing and functional genomics tools. To date, a number of critical genes involved in abiotic stress tolerance have been identified and validated, which are generally classified into two types: functional genes and regulatory genes. ${ }^{3}$ A major limitation is due to dependence on a number of physiological traits and each under multigenic control, which arises complexity of tolerance to abiotic stresses. Complexity in stress tolerance traits causes marginal success in conventional breeding approaches hence, the transgenic approach is now being commonly used to breed stress-tolerant crops. Although, there are several stress tolerance genes have been identified and made the transgenic plants, but they can only useful to combat one or two distinct stresses. The production of crops with multiple genetically engineered traits may seem a logical step, as more locally adapted transgenic varieties become available and accepted in the market, these will become the source material into which novel genes will be incorporated. Nevertheless, some difficulties like trait silencing due to similarities between transgene cassettes or epistatic interactions between transgenes, can arise if too many transgenes are incorporated into a single variety, and such effects may be cumulative over successive generations. Hence, rather than manipulation of the single functional gene, engineering certain regulatory genes have emerged as an effective strategy for controlling the expression of many stressresponsive genes. Transcription factors (TFs) are good candidates for genetic engineering to breed stress-tolerant crop because of their role as master regulators of many stress-responsive genes. Many TFs belonging to families AP2/EREBP, MYB, WRKY, NAC, bZIP have been found to be involved in various abiotic stresses and some TF genes have also been engineered to improve stress tolerance in the model and crop plants. ${ }^{2}$

The development of crop varieties (transgenic or non-transgenic) which can tolerate abiotic stresses would be of major benefit to agriculture in cropping regions where abiotic stresses are a chronic problem. No transgenic crop variety tolerance to abiotic stress has so far been reported to be released for cultivation. However, some transgenic varieties exhibiting tolerance to a range of abiotic stresses underwent field testing in Bolivia (a frost tolerant potato variety), China (a cold tolerant tomato), Egypt (a salt tolerant wheat variety),
Volume 4 Issue 4 - 2016

\author{
Verma AK, Singh Deepti \\ Department of Biochemistry, GB Pant University of Agriculture \\ and Technology, India
}

Correspondence: Verma AK, Department of Biochemistry, College of Basic Sciences and Humanities, GB Pant University of Agriculture and Technology, Pantnagar- 263145 Uttarakhand, India, Tel +9I-0594-422-333-I0, Fax +9I-05944-233473, Email akv72@rediffmail.com

Received:September 14, 2016 | Published: September 14 2016

India (moisture stress tolerant Brassica variety) and Thailand (salt tolerant and drought tolerant rice varieties). While, a number of research initiatives at the laboratory stage. Most of the R\&D activities on the development of abiotic stress tolerant crops are being carried out in six countries of the Asian region, namely Bangladesh, China, India, Indonesia, Pakistan, and Thailand. China is relatively active in this area and has reported preliminary successes with rice, maize, and sorghum tolerant to high salt concentrations. Transgenic studies on salt resistant crop development are also being undertaken on rice in Bangladesh, Brazil, India and Pakistan, and on tobacco in Argentina. Despite the effects of drought on crop production, very little transgenic research on drought resistance is reportedly being carried out in the five regions. Drought-tolerant rice has been field tested in Thailand. The rest of the activities in this area are mainly at the laboratory stage, with work on sugar cane (Indonesia), rice (China and Indonesia), and groundnut (South Africa). ${ }^{4}$

Abiotic stress response in plants is an enormously complex process due to huge gene families' involvement and the multifaceted interactions between TFs and cis-elements on the promoters of target genes. However, one transcription factor may regulate a vast array of target genes with the corresponding cis-elements on the promoters, whereas one gene with several types of cis-elements may be regulated by different families of TFs. Thus, the stress-responsive TFs not only function independently, but also crosstalk between each other in response to various abiotic stress responses, which indicates the complexity of signaling networks involved in plant stress responses. In future, it is essential to identify multiple stress-responsive TF genes and compare their expression patterns followed by identification of commonly regulated genes responsive for universal stress responses. ${ }^{5}$ Transgenic approach after genetic manipulation of these identified genes should be a more powerful approach for improving plant tolerance to multiple stresses than manipulation of individual functional genes. Furthermore, the critical field trials are required to evaluate the transgenic plants, especially focusing on their growth and tolerance in the whole life period. That should a necessary step in many strategies to develop stress-tolerant crops. It is also desirable to identify a complete regulatory mechanism involved in plant abiotic stress responses, which will be helpful to obtain the promising TF 
genes for breeding multiple abiotic stress-tolerant crops with better yields and qualities.

The overall impact of the opinion to discuss some recent issue which could stimulate many more researchers to utilize the transgenic approach by using the TF to combat the complex problem abiotic stress. This can only be achieved with a better-understood connection of complimenting areas of physiology and biochemistry to the genetic component that controls them.

\section{Acknowledgements}

\section{None.}

\section{Conflict of interest}

The author declares no conflict of interest.

\section{References}

1. Ribant JM, Banziger M, Hoisington D. Genetic dissection and plant improvement under abiotic stress conditions: drought tolerance in maize as an example. JIRCAS Working Report. 2002;2002:85-92.

2. Wang $\mathrm{H}$, Wang $\mathrm{H}$, Shao $\mathrm{H}$, et al. Recent advances in utilizing transcription factors to improve plant abiotic stress tolerance by transgenic technology. Front Plant Sci. 2016;7:67.

3. Shinozaki K, Yamaguchi-Shinozakiy K, Sekiz M. Regulatory network of gene expression in the drought and cold stress responses. Curr Opin Plant Biol. 2003;6(5):410-417.

4. Dhlamin Z, Spillane C, Moss JP, et al. Status of research and application of crop biotechnology in developing countries. Food and Agriculture Organization of the United Nation Rome. 2005. p. 35-36.

5. Shao H, Wang H, Tang X. NAC transcription factors in plant multiple abiotic stress responses: progress and prospects. Front Plant Sci. 2015;6:902. 